\title{
An Elicitation Procedure for the Generalized Trapezoidal Distribution with a Uniform Central Stage
}

Johan René van Dorp ${ }^{1}$, The George Washington University, USA

Salvador Cruz Rambaud ${ }^{2}$, University of Almería, Spain

José García Pérez ${ }^{3}$, University of Almería, Spain

Rafael Herrerías Pleguezuelo ${ }^{4}$, University of Granada, Spain

\begin{abstract}
Recent advances in computation technology for decision/simulation and uncertainty analyses have revived interest in the the triangular distribution and its use to describe uncertainty of bounded input phenomena. The trapezoidal distribution, explicitly suggested by Pouliquen (1970) in the framework of risk and uncertainty analysis, is a generalization of the triangular distribution that allows for the specification of the modal value by means of a range of values rather than a single point estimate. While the trapezoidal and the triangular distributions are restricted to linear geometric forms in the successive stages of the distribution, the generalized trapezoidal (GT) distribution introduced by van Dorp and Kotz (2003) allows for a non-linear behavior at its tails and a linear incline (or decline) in the central stage. In this paper we shall develop two novel elicitation procedures for the parameters of a special case of the GT family by restricting ourselves to a uniform (horizontal) central stage in accordance with the central stage of the original trapezoidal distribution.
\end{abstract}

\footnotetext{
${ }^{1}$ Corresponding Author. Department of Engineering Management and Systems Engineering, School of Engineering and Applied Science, The George Washington University, 1776 G Street, N.W., Suite 110, Washington D.C. 20052. Phone: 202-994-6638, Fax: 202-994-0245, E-mail: dorpjr@gwu.edu.

${ }^{2}$ Departamento de Dirección y Gestión de Empresas. University of Almería (Spain). E-mail: scruz@ual.es ${ }^{3}$ Departamento de Economía Aplicada. University of Almería (Spain). E-mail: jgarcia@ual.es ${ }^{4}$ Departamento de Métodos Cuantitativos para la Economía y la Empresa. University of Granada (Spain). E-mail: rherreri@platon.ugr.es
} 


\section{Introduction}

In a recent survey paper a leading Bayesian statistician, O'Hagan (2006), explicitly mentions a need for advances in elicitation techniques for prior distributions in Bayesian Analyses, but also acknowledges the importance of their development for those areas where the elicited distribution can not be combined with evidence from data, because the expert opinion is essentially all the available knowledge. Garthwaite, Kadane and O'Hagan (2005) provide a comprehensive review on the topic of eliciting probability distributions dealing with a wide variety of topics, such as, e.g., the elicitation process, heuristics and biases, fitting distributions to an expert's summaries, expert calibration and group elicitation methods. We encourage the reader to review the bibliography of Garthwaite, Kadane and O'Hagan (2005) which is impressive and contains over 100 references. Merkhofer's (1987) paper, not listed in Garthwaite, Kadane and O'Hagan (2005), also provides a practical perspective in using judgmental probability distributions in real-world problems.

The topic of this paper deals with fitting a specific parametric distribution to a set of summaries elicited from an expert. Experts are traditionally classified into two, usually unrelated, groups: 1) substantive experts (also known as technical experts or domain experts) who are knowledgeable about the subject matter at hand and 2) normative experts mainly possessing knowledge of the appropriate quantitative analysis techniques (see, e.g., DeWispelare et al. (1995) and Pulkkinen and Simola (2000)). In the absence of data and in the context of decision/simulation and uncertainty analyses, substantive experts are used (primarily by necessity) to specify input distributions.

Advances in decision/simulation and uncertainty analysis methodology and their penetration into applied sciences and engineering during the last several decades (recall - by now standard tools such as Decision Tool Suite by the Palisade Corporation, Crystal Ball by Decision Engineering, and ARENA by Rockwell Software) have reinvigorated the use of distributions with bounded support (that were not initially popular options). Integration of graphically interactive and statistical procedures for bounded input distribution modeling has become a topic of research (see, e.g., DeBrota et al. (1989), AbouRizk et al. (1992) and Wagner and Wilson $(1995,1996))$ in order to facilitate their elicitation by experts. AbouRizk et al. (1992) have developed software with a graphical 
user interface (GUI) to ease fitting of beta distributions using a variety of methods and DeBrota et al. (1989) have developed software for fitting bounded Johnson $\mathrm{S}_{B}$ distributions. Wagner and Wilson (1995, 1996) introduced univariate Bézier distributions (or curves), which are a variant of spline functions, and the software tool PRIME with a GUI to specify them. All these methods involve the requirement of specifying the lower and upper bounds of the distribution's support. While the system of Bézier distributions allows for great flexibility in input distribution modeling for stochastic simulations, Wagner and Wilson (1996) point out that random variate generation from a Bézier distribution is at present computationally inefficient since its $F^{-1}$ (the inverse cumulative distribution function (cdf)) cannot be expressed in a closed form. The same applies for the beta or Johnson $\mathrm{S}_{B}$ distributions. Fortunately, triangular, trapezoidal and generalized trapezoidal distributions do have closed form cdf's.

Trapezoidal distributions have been advocated for use in risk analysis problems, initially by Pouliquen (1970) and more recently by Herrerías and Calvete (1987), Herrerías (1989), Powell and Wilson (1997) and Garvey (2000). Other applications of trapezoidal distributions are prominent in applied physics problems (see, e.g., Davis and Sorenson (1969), Nakao and Iwaki (2000), Sentenac et al. (2000)) and medical ones, specifically in the screening and detection of cancer (see, e.g., Flehinger and Kimmel (1987), Brown (1999) and Kimmel and Gorlova (2003)). Trapezoidal distributions have also been used as membership functions in fuzzy set theory (see, e.g., Chen and Hwang (1992) and Bardosi and Fodor (2004)).

Figure 1A plots a trapezoidal probability density function (pdf) suggested by Pouliquen (1970) with the boundary parameters $a=0, b=0.3, c=0.5$ and $d=1$. The trapezoidal pdf depicted in Figure $1 \mathrm{~A}$ is a generalization of the "classical" triangular distribution dating back as far as Simpson ${ }^{5}$ $(1755,1757)$. Analogously to the triangular distribution, the trapezoidal distribution is appealing in practice mainly due to the ease of the physical interpretation of its parameters $a, b, c$ and $d$. This would allow for their straightforward elicitation via a substantive expert knowledgeable about an

${ }^{5}$ Thomas Simpson (1710-1761) a prolific writer of mathematical textbooks and able teacher at the Royal Military Academy in Wolwich (England) made original and important contributions to statistics and actuarial sciences. 
uncertain phenomenon represented by the distribution. However, the requirement of specifying the bounds when using input distributions with bounded support in decision/simulation and uncertainty analyses poses some challenges. Although the use of bounded distributions in the absence of data is by now prevalent, the fact that the lower and upper bounds of an uncertain phenomenon as a rule fall outside of the accumulated experience of a substantive expert (see, e.g., Selvidge (1980), Davidson and Cooper (1980), Alpert and Raiffa (1982), Keefer and Verdini (1993)) is rarely acknowledged. Instead these authors suggest the elicitation of lower and upper quantiles instead. Keefer and Bodily (1983) solved for the lower and upper bounds of a triangular distribution in the case when a point estimate for its mode is also available. Kotz and Van Dorp (2006) extended Keefer and Bodily's (1983) procedure for Two-Sided Power (TSP) distributions that are generalizations of triangular distribution allowing for non-linear behavior in the two tails.

Van Dorp and Kotz (2003) provide the probability density function (pdf) of the Generalized Trapezoidal (GT) distribution with parameters $\alpha, a, b, c, d, m$ and $n$ given by

$$
f_{X}(x \mid \Theta)=\mathcal{C}(\Theta) \times \begin{cases}\alpha\left(\frac{x-a}{b-a}\right)^{m-1}, & \text { for } a \leq x<b \\ (1-\alpha)\left(\frac{x-b}{c-b}\right)+\alpha, & \text { for } b \leq x<c \\ \left(\frac{d-x}{d-c}\right)^{n-1}, & \text { for } c \leq x<d\end{cases}
$$

where the parameter vector $\Theta=\{a, b, c, d, m, n, \alpha\}, a<b<c<d$ and $m, n, \alpha>0$ and the normalizing constant is

$$
\mathcal{C}(\Theta)=\frac{2 m n}{2 \alpha(b-a) n+(\alpha+1)(c-b) m n+2(d-c) m} .
$$

The parameter $\alpha=f_{X}(b \mid \Theta) / f_{X}(c \mid \Theta)$ and is referred to as a boundary ratio parameter. The generalization (1) allows for non-linear behavior in the tails of the pdf via the tail parameters $m$ and $n$ and a linear incline (or decline) of the pdf in the central stage by setting the boundary ratio parameter $\alpha \neq 1$. It possesses a closed form cdf. Figure 1B plots a generalization of the pdf presented in Figure 1A with the same boundary parameters $a, b, c$ and $d$ and the additional parameter values $\alpha=1 \frac{1}{4}, m=3$ and $n=5$. By substituting $n=m=2$ and $\alpha=1$ in $(1)$ the pdf 
(1) reduces to the "classical trapezoidal" pdf. Apparently, elicitation procedures for the additional parameters of the GT distribution have not so far been developed. These type of procedures may be useful for its application in problems of decision/risk and uncertainty analysis.
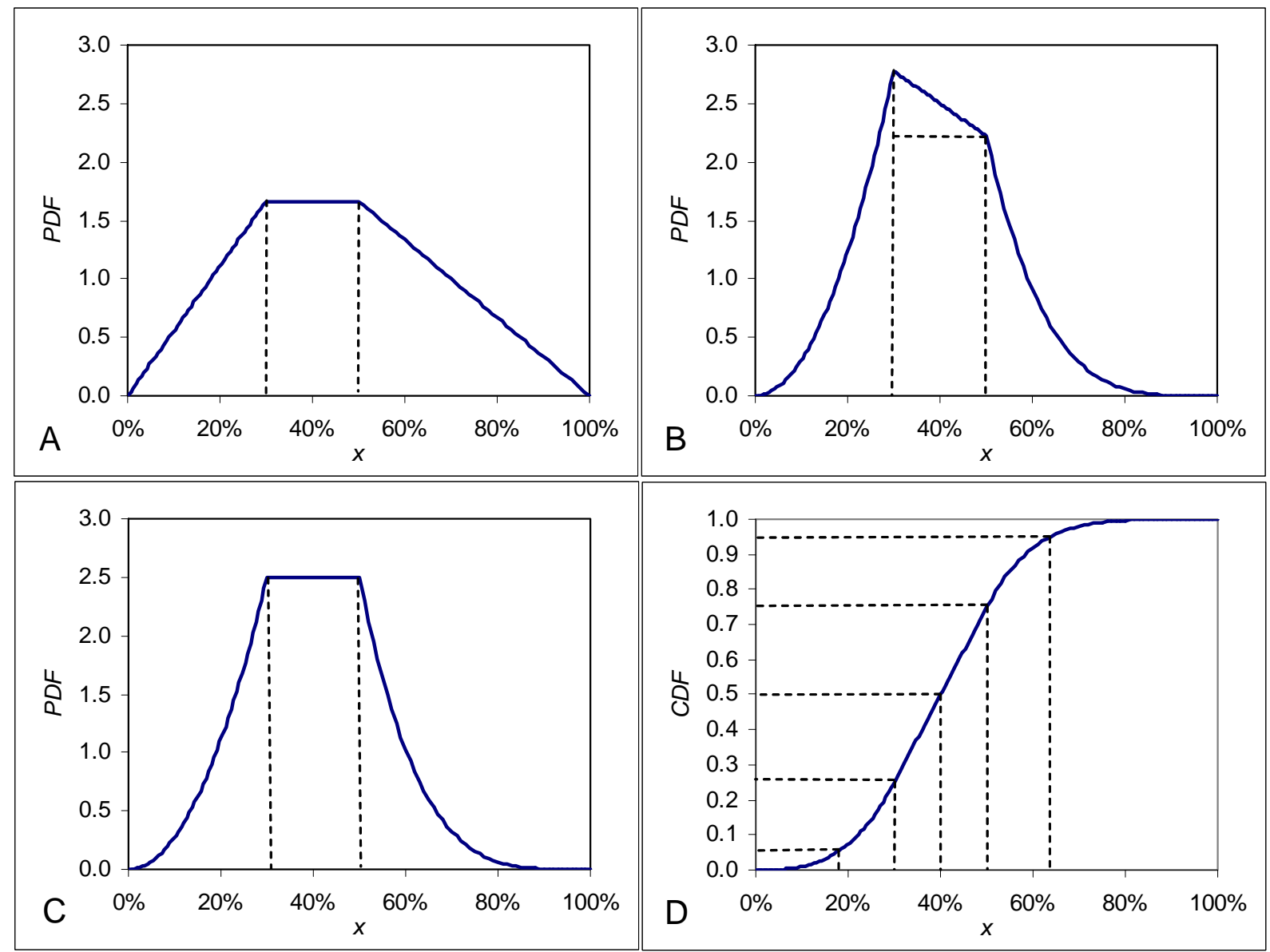

Fig. 1. Generalized trapezoidal (GT) densities with common boundary parameters $a=0, b=0.3, c=0.5$ and $d=1$; A: Original trapezoidal pdf with $m=2, n=2, \alpha=1$, B: GT pdf with $m=3, n=5$, and $\alpha=1 \frac{1}{4}$, C: GT Uniform pdf with $m=3, n=5$, and $\alpha=1$, D: GTU cdf with $m=3, n=5$, and $\alpha=1$.

We shall restrict ourselves in this paper to the analysis of GT distributions with a uniform central stage. This is achieved by setting $\alpha=1$ in (1) and (2) and referring to it as Generalized Trapezoidal-Uniform (GTU) distributions it has the pdf: 


$$
f_{X}(x \mid \Phi)=\mathcal{C}(\Phi) \times \begin{cases}\left(\frac{x-a}{b-a}\right)^{m-1}, & \text { for } a \leq x<b \\ 1, & \text { for } b \leq x<c \\ \left(\frac{d-x}{d-c}\right)^{n-1}, & \text { for } c \leq x<d\end{cases}
$$

where the parameter vector $\Phi=\{a, b, c, d, m, n\}, a<b<c<d$ and $m, n>0$ and the normalizing constant $\mathcal{C}(\Phi)$ is given by

$$
\mathcal{C}(\Phi)=\frac{m n}{(b-a) n+(c-b) m n+(d-c) m} .
$$

Figure 1C (Figure 1D) displays the GTU pdf (cdf) of Figure 1B after substituting $\alpha=1$ instead of $\alpha=1 \frac{1}{4}$. Note that the modal value of the GTU pdf $(3)$ is attained for all values in the central stage $[b, c]$. Hence, similarly to the original trapezoidal distribution (Figure 1A) one may directly elicit this modal range by means of a substantive expert (who may be more "comfortable" here, being relieved of providing a fixed point estimate for the modal value as required for a triangular distribution). Unfortunately, this is not plausible for the more general GT family (1) involving the boundary ratio parameter $\alpha$ (see, e.g., van Dorp and Kotz (2003)).

In the remainder of this paper we shall propose two elicitation procedures for the parameters of GTU distributions (3). In Section 2, the first method will be presented assuming that the boundary parameters $a$ and $d$ are known due to natural boundary constraints such as, for example, a percentage of a total population or a probability having a natural support $[0,1]$. After eliciting the central stage bounds $b$ and $c$, the tail parameters $m$ and $n$ will indirectly be elicited from a substantive expert following the fixed interval method mentioned in Garthwaite, Kadane and O'Hagan (2005). The second method, to be discussed in Section 3, deals with unknown boundary parameters $a$ and $d$ and elicits also a lower $a_{p}<b$ and upper quantiles $d_{r}>c$ which are used to solve for these lower $a$ and the upper $d$ bounds ( $p$ and $r$ are usually assumed to be equal to 0.05 and 0.95 or 0.10 and 0.90 , respectively). 


\section{Indirect elicitation of tail parameters with fixed lower and upper bounds}

Mixing distributions is common practice in dealing with e.g. Phase-Type, Erlang, Poisson and Normal distributions (see, e.g., Johnson and Taaffe (1991) and Karlis and Xekalaki (1999)). The pdf (3) may be expressed as a mixture (see, e.g., van Dorp and Kotz (2003)) involving three densities $f_{X_{1}}, f_{X_{2}}, f_{X_{3}}$ with bounded support, such that

$$
f_{X}(x \mid \Phi)=\sum_{i=1}^{3} \pi_{i} f_{X_{i}}(x \mid \Phi), \quad \sum_{i=1}^{3} \pi_{i}=1, \pi_{i}>0,
$$

where as above $\Phi=\{a, b, c, d, m, n\}$. Here

$$
\begin{aligned}
& f_{X_{1}}(x \mid \Phi)=f_{X_{1}}(x \mid a, b, m)=\left(\frac{m}{b-a}\right)\left(\frac{x-a}{b-a}\right)^{m-1}, \\
& a \leq x<b, m>0 \\
& \quad f_{X_{2}}(x \mid \Phi)=f_{X_{2}}(x \mid b, c)=\frac{1}{c-b}, b \leq x \leq c
\end{aligned}
$$

and

$$
\begin{aligned}
& f_{X_{3}}(x \mid \Phi)=f_{X_{3}}(x \mid c, d, n)=\left(\frac{n}{d-c}\right)\left(\frac{d-x}{d-c}\right)^{n-1} \\
& c \leq x<d, n>0 .
\end{aligned}
$$

The mixture probabilities $\pi_{i}, i=1,2,3$, are

$$
\left\{\begin{array}{l}
\pi_{1}=\mathcal{C}(\Phi)(b-a) / m \\
\pi_{2}=\mathcal{C}(\Phi)(c-b) \\
\pi_{3}=\mathcal{C}(\Phi)(d-c) / n
\end{array}\right.
$$

where $\mathcal{C}(\Phi)$ is given by (4). Observe that the mixture weight of the first stage $\pi_{1}$ decreases as its tail parameter $m$ increases. A similar observation can be made for the third stage with obvious modification.

After some algebraic manipulations we derive from (5) - (9)

$$
E[X \mid \Phi]=\mathcal{C}(\Phi) \times\left[\frac{a+m b}{m(m+1)}(b-a)+\frac{b+c}{2}(c-b)+\frac{n c+d}{n(n+1)}(d-c)\right]
$$

and 


$$
\begin{aligned}
E\left[X^{2} \mid \Phi\right]=\mathcal{C}(\Phi) \times\{ & {\left[\frac{a^{2}}{m}+\frac{2 a(b-a)}{m+1}+\frac{(b-a)^{2}}{m+2}\right](b-a)+} \\
& \left.\left.\frac{b^{2}+b c+c^{2}}{3}(c-b)+\left[\frac{d^{2}}{n}-\frac{2 d(d-c)}{n+1}+\frac{(d-c)^{2}}{n+2}\right](d-c)\right]\right\} .
\end{aligned}
$$

Note that in expressions (10) and (11) the second factor is a weighted sum of the support widths $(b-a),(c-b)$ and $(d-c)$, where the weights of the first and the third terms are also, but not solely, determined by the tail parameters $m$ and $n$, respectively. Expressions (10) and (11) allow for straightforward evaluation of the variance $\operatorname{Var}[X]=E\left[X^{2} \mid \Phi\right]-E^{2}[X \mid \Phi]$.

Finally, from (5) - (9) one straightforwardly obtains the following convenient form for the cdf of $(5)$ (equivalently $(3)$ )

$$
F_{X}(x \mid \Phi)= \begin{cases}\pi_{1}\left(\frac{x-a}{b-a}\right)^{m}, & a \leq x \leq b \\ \pi_{1}+\pi_{2} \frac{x-b}{c-b}, & b<x \leq c \\ 1-\pi_{3}\left(\frac{d-x}{d-c}\right)^{n}, & c<x \leq d\end{cases}
$$

and quantile function (or inverse cdf $F^{-1}$ ) :

$$
F_{X}^{-1}(y \mid \Phi)= \begin{cases}a+\left(\frac{y}{\pi_{1}}\right)^{1 / m}(b-a), & 0 \leq y \leq \pi_{1}, \\ b+\frac{y-\pi_{1}}{\pi_{2}}(c-b), & \pi_{1}<y \leq 1-\pi_{3}, \\ d-\left(\frac{1-y}{\pi_{3}}\right)^{1 / n}(d-c), & 1-\pi_{3}<y \leq 1,\end{cases}
$$

where the mixture probabilities $\pi_{1}, \pi_{2}$ and $\pi_{3}$ are given by $(9)$. Note that the quantile function $F_{X}^{-1}(y \mid \Phi)$ involves convex combinations of the boundaries of each stage reminiscent of the quantile function of a uniform distribution. This allows for a straightforward implementation of a sampling procedure from GTU distributions using a pseudo-random number generator (see, e.g. Banks et al. (2005)).

Assume now that the parameters $a$ and $d$ are known and that the modal range $[b, c]$ has been directly elicited from a substantive expert. We shall now proceed using the fixed interval method mentioned in Garthwaite, Kadane and O'Hagan (2005). Namely, we suggest eliciting the relative likelihoods $\pi_{2} / \pi_{1}$ and $\pi_{2} / \pi_{3}$ (or their reciprocals) of the uncertain quantity at hand falling in the 
central stage $[b, c]$ relative to the tails $[a, b)$ and $(c, d]$, respectively. Next, we directly solve for the tail parameters $m$ and $n$ utilizing relationships:

$$
\left\{\begin{array} { l } 
{ \frac { \pi _ { 2 } } { \pi _ { 1 } } = m \frac { c - b } { b - a } , } \\
{ \frac { \pi _ { 2 } } { \pi _ { 3 } } = n \frac { c - b } { d - c } . }
\end{array} \Leftrightarrow \left\{\begin{array}{l}
m=\frac{\pi_{2}(b-a)}{\pi_{1}(c-b)}, \\
n=\frac{\pi_{2}(d-c)}{\pi_{3}(c-b)},
\end{array}\right.\right.
$$

which immediately follow from (9). Observe that expression (14) implies that the ratio of the central stage and first (third) stage probability equals $m(n)$ times the ratio of the widths of their corresponding supports.

As an example of the above elicitation procedure, suppose a decision maker Jasmine Devereaux (J.D.) whishes to estimate her market share of a new clothing line in the upcoming season. J.D. is convinced it will most likely fall between $30 \%$ and $50 \%$. Moreover, she believes it to be twice as likely for this market share to fall within the range $[30 \%, 50 \%]$ as compared to being either less than $30 \%$ or more than $50 \%$. Figure 1C (Figure 1D) depicts a GTU pdf (cdf) with natural support $[0,100 \%]$ that is consistent with J.D. degree of beliefs. From her statements we deduce it is twice as likely for her market share to fall within the modal range $[30 \%, 50 \%]$ as compared to the tails $[0,30 \%)$ and $(50 \%, 100 \%]$. Hence, $\pi_{2} / \pi_{1}=\pi_{2} / \pi_{3}=2$, yielding $\pi_{2}=0.5$ and $\pi_{1}=\pi_{3}=0.25$.

The parameter values $m=3$ and $n=5$ in Figure $1 \mathrm{C}$ and $\mathrm{D}$ now follow directly from (14). J.D. evaluates from (10) and (11) a mean market share of $40.2 \%$ and a market share standard deviation equal to $14.1 \%$. She utilizes $(13)$ to evaluate the quantiles $x_{0.05} \approx 17.5 \%, x_{0.50} \approx 40 \%$ and $x_{0.95} \approx 63.8 \%$ (see Figure $1 \mathrm{D}$ ). In other words, she believes her median market share is virtually identical to her mean market share and its subjective $90 \%$ credibility interval equals $(17.5 \%$, $63.8 \%)$.

\section{Indirect elicitation of tail parameters and lower and upper bounds}

The elicitation of lower and upper quantiles for a bounded uncertain quantity adheres to the prevailing view that lower and upper bounds of an uncertain phenomenon as a rule fall outside of the accumulated experience of a substantive expert (see, e.g., Selvidge (1980), Davidson and Cooper 
(1980), Alpert and Raiffa (1982), Keefer and Verdini (1993)). Forcing a substantive expert in such a scenario to provide strict lower and upper bound estimates may lead to a misrepresentation of uncertainty. The elicitation of the quantiles $a_{0.10}$ and $d_{0.90}$ was suggested by Keefer and Verdini (1993). Instead of specifying the values $p=0.10$ and $r=0.90$ an alternative procedure could be to request the substantive expert to specify some other quantile levels $p$ and $r$ that he/she is comfortable with. Thus we shall assume here that the lower and upper bound parameters $a$ and $d$ and tail parameters $m$ and $n$ are unknown.

Moreover, we shall assume that the bound parameters $a, d$ and tail parameters $m$ and $n$ need to be determined from $(i)$ a directly elicited modal range $[b, c]$, (ii) the relative likelihoods $\pi_{2} / \pi_{1}$ and $\pi_{2} / \pi_{3}$, and (iii) a lower $a_{p}<b$ and upper $d_{r}>c$ quantiles. The ratio $\pi_{2} / \pi_{1}\left(\pi_{2} / \pi_{3}\right)$ may be elicited here by eliciting the likelihood of the central stage $[b, c]$ relative to the uncertain quantity being less (larger) than the lower bound $b$ (upper bound $c$ ) of this central stage. The probabilities associated with each interval are then obtained utilizing that their sum must be 1 (see, Garthwaite, Kadane and O'Hagan (2005)). From expression (14), we can immediately write the lower $a$ and the upper $d$ bounds as linear functions of the tails parameters $m$ and $n$, respectively. Specifically,

$$
\left\{\begin{array}{l}
a=b-\frac{\pi_{1}(c-b)}{\pi_{2}} m \equiv a^{*}(m), \\
d=c+\frac{\pi_{3}(c-b)}{\pi_{2}} n \equiv d^{*}(n) .
\end{array}\right.
$$

(Notation $a^{*}(m)\left[d^{*}(n)\right]$ for $a[d]$ emphasizes their dependence on $\left.m[n].\right)$ Note that the expressions (15) do not involve the lower $a_{p}$ and upper $d_{r}$ quantiles, but result directly from the relations (14) linking the probability in each stage of the GTU distribution with the width of the support of each stage via the tail parameters $m$ and $n$.

In the Subsections 3.1 and 3.2 we shall derive two additional functions such that $a \equiv \widetilde{a}(m)$ and $d \equiv \widetilde{d}(n)$ that describe a non-linear relationship between the tails parameters $m$ and $n$ and the lower $a$ and upper $d$ bounds. These relationships do involve $a_{p}$ and $d_{r}$ and, under certain uniqueness conditions, we may solve for $m(n)$ by setting $a^{*}(m)=\widetilde{a}(m)$ (by setting $\left.d^{*}(n)=\widetilde{d}(n)\right)$ 


\subsection{Solving for the left tail parameter $m$ and the lower bound $a$}

From the expressions for the GTU cdf (12) and the definition of a lower quantile $a_{p}<b$ we obtain (by substitution) that

$$
F_{X}\left(a_{p} \mid \Phi\right)=\pi_{1}\left(\frac{a_{p}-a}{b-a}\right)^{m}=p \Leftrightarrow a=a_{p}-\frac{\lambda\left(m, p, \pi_{1}\right)}{1-\lambda\left(m, p, \pi_{1}\right)}\left(b-a_{p}\right) \equiv \widetilde{a}(m),
$$

where $p<\pi_{1}$ and

$$
0<\lambda\left(m, p, \pi_{1}\right)=\left(p / \pi_{1}\right)^{1 / m}<1
$$

Note that the left hand side (LHS) of expression (16) links the first stage probability $\pi_{1}$ to the quantile level $p$, the width of the support of the first stage $(b-a)$ and the distance $\left(a_{p}-a\right)$ from the lower bound to the lower quantile $a_{p}$.

Note that both $\widetilde{a}(m)$ and $a^{*}(m)$ are just expressions for the same parameter $a$ in different situations. We may now solve for a tail parameter $m$ satisfying the lower quantile constraint (14) by setting

$$
\widetilde{a}(m)=a^{*}(m)
$$

where $a^{*}(m)$ is the linear function defined by (15). In the appendix we shall prove that the LHS lower bound function $\widetilde{a}(m)$ in $(18)$ is concave, strictly decreasing with the asymptote

$$
\mathcal{A}(m)=\frac{b-a_{p}}{\log \left(\frac{p}{\pi_{1}}\right)} m+\frac{a_{p}+b}{2} .
$$

From $\widetilde{a}(m)<a_{p}$ for all $m>0$ (see $\left.(16)\right)$ and the properties of the function $\widetilde{a}(m)$, it immediately follows that the number of solutions of equation (18) equals that of the equation

$$
\mathcal{A}(m)=a^{*}(m)
$$

However, this number can be at most one, since both functions in (20) are linear. From,

$$
\frac{a_{p}+b}{2}=\mathcal{A}(0)<a^{*}(0)=b \text {. }
$$


it next follows that a unique solution for the tail parameter $m$ exists for equation (18) iff when the slope of the asymptote $\mathcal{A}(m)$ is less steep than that of the linear function $a^{*}(m)$ defined by $(15)$, i.e.

$$
\frac{b-a_{p}}{\log \left(\frac{p}{\pi_{1}}\right)}<-\frac{\pi_{1}(c-b)}{\pi_{2}} \Leftrightarrow b-\xi(c-b)<a_{p}
$$

where

$$
\xi=\frac{\pi_{1}}{\pi_{2}} \log \left(\frac{\pi_{1}}{p}\right)>0
$$

Hence, condition (22) determines both the existence and uniqueness of a solution for equation (18).

Figure 2A depicts both functions $a^{*}(m), \widetilde{a}(m)$ and the asymptote $\mathcal{A}(m)$ for the case that $b=80, c=100, \pi_{2} / \pi_{1}=1.5$ and $\pi_{2} / \pi_{3}=1$ and lower quantile $a_{0.10}=71$. We have from $\pi_{2} / \pi_{1}=1.5$ and $\pi_{2} / \pi_{3}=1$ that

$$
\pi_{1}=\frac{1}{4}, \pi_{2}=\pi_{3}=\frac{3}{8}
$$

For the data in Figure 2A the condition (22) reduces to

$$
80-13 \frac{1}{3} \log (2.5) \approx 67.783<a_{0.10}=71 .
$$

Hence, a unique solution of equation (18) exists for the data in Figure 2A. Solving for $m$ using a standard root finding algorithm yields

$$
m=1.419
$$

and substituting $m=1.419$ in either $\widetilde{a}(m)(16)$ or $a^{*}(m)(15)$ yields the lower bound

$$
a=\widetilde{a}(m)=a^{*}(m) \approx 61.085 .
$$

Figure $2 \mathrm{~B}$ plots the functions $\tilde{a}(m), a^{*}(m)$ and the asymptote $\mathcal{A}(m)$ for the boundary case $(22)$ $a_{0.10} \approx 67.783$. Observe that in this case no solution for equations (18) and (20) exists. 
From (22) and (23) we conclude that the pre-assigned quantile level $p$ provides a lower threshold for the quantile $a_{p}$ defined (22). This threshold is just a function of the width of the central stage $(c-b)$, its probability $\pi_{2}$, the first stage probability $\pi_{1}$ and the quantile level $p$. In case a substantive expert specifies a set of values for $a_{p}, \pi_{2}, \pi_{1}$ and $b$ and $c$ for which the condition $(22)$ is not met, he/she may be given the option to revise his/her assessments utilizing the threshold value $b-\xi(c-b)$ in $(22)$ as feedback. The use of feedback to enhance consistency in an expert's judgment is quite common (see, e.g., Denham and Mengersen (2007)).

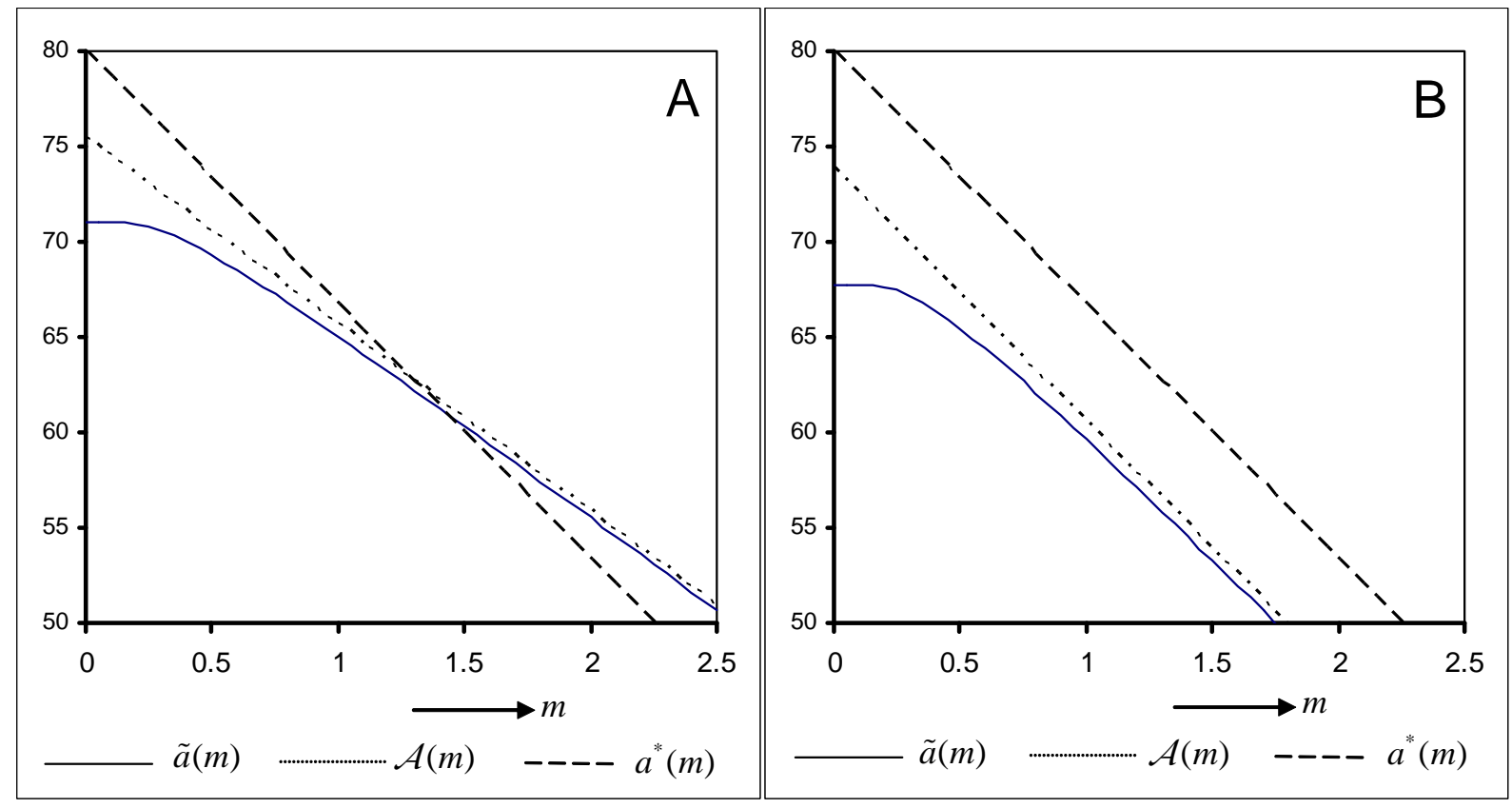

Fig. 2. Lower bound functions $a^{*}(m)(15)$ and $\widetilde{a}(m)(16)$ with its asymptote $\mathcal{A}(m)$ (19) for the data $b=80, c=100, \pi_{1}=25 \%, \pi_{2}=37.5 \%$; A: lower quantile $a_{0.10}=71$, B: lower quantile $a_{0.10} \approx 67.783$.

\subsection{Solving for the right tail parameter $n$ and the upper bound}

After fully digesting the derivations in subsection 3.1 this subsection is straightforward. From the expression for the GTU $\operatorname{cdf}(12)$ and the definition of the upper quantile $d_{r}>c$ we obtain that

$$
F_{X}\left(d_{r} \mid \Phi\right)=1-\pi_{3}\left(\frac{d-x}{d-c}\right)^{n}=r \Leftrightarrow d=d_{r}+\frac{\mu\left(n, r, \pi_{3}\right)}{1-\mu\left(n, r, \pi_{3}\right)}\left(d_{r}-c\right) \equiv \widetilde{d}(n)
$$


where $\pi_{3}>1-r$ and

$$
0<\mu\left(n, r, \pi_{3}\right)=\left\{(1-r) / \pi_{3}\right\}^{1 / n}<1
$$

Analogously as in Subsection 3.1, we may solve for the tail parameter $n$ satisfying the upper quantile constraint $(28)$ by setting

$$
\widetilde{d}(n)=d^{*}(n)
$$

where $d^{*}(n)$ is the linear function defined by $(15)$. The following properties of $\widetilde{d}(n)$ are derived in the appendix: $\widetilde{d}(0)=d_{r}, \widetilde{d}(n)$ is strictly increasing, is convex and possesses the asymptote

$$
\mathcal{D}(n)=\frac{c-d_{r}}{\log \left(\frac{1-r}{\pi_{3}}\right)} n+\frac{c+d_{r}}{2} .
$$

A unique solution to equation (30) exists iff

$$
d_{r}<c+\psi(c-b)
$$

where

$$
\psi=\frac{\pi_{3}}{\pi_{2}} \log \left(\frac{\pi_{3}}{1-r}\right)>0
$$

Figure $3 \mathrm{~A}$ depicts both functions $\widetilde{d}(n)$ defined by $(28), d^{*}(n)$ defined by $(15)$ and the asymptote $\mathcal{D}(n)$ defined by $(32)$ for the case that $b=80, c=100, \pi_{2} / \pi_{1}=1.5$ and $\pi_{2} / \pi_{3}=1$ and upper quantile $d_{0.90}=121$. For this data a unique solution of $(30)$ exists since the RHS threshold of $(33) c+\psi(c-b)$ equals

$$
100+20 \log (3.75) \approx 126.425>d_{0.90}=121 .
$$

Solving for $n$ using a standard root-finding algorithm yields

$$
n=2.757
$$

and substituting $n=2.757$ in either $\widetilde{d}(n)$ defined by (28) or $d^{*}(n)$ defined by (15) yields the upper bound

$$
d=\widetilde{d}(n)=d^{*}(m) \approx 155.135
$$


Figure $3 \mathrm{~B}$ plots the functions $\widetilde{d}(m), d^{*}(m)$ and the asymptote $\mathcal{D}(n)$ for the case of the LHS boundary $d_{0.90} \approx 126.425$ given by $(35)$. Observe that here no solution exists for equation $(30)$.

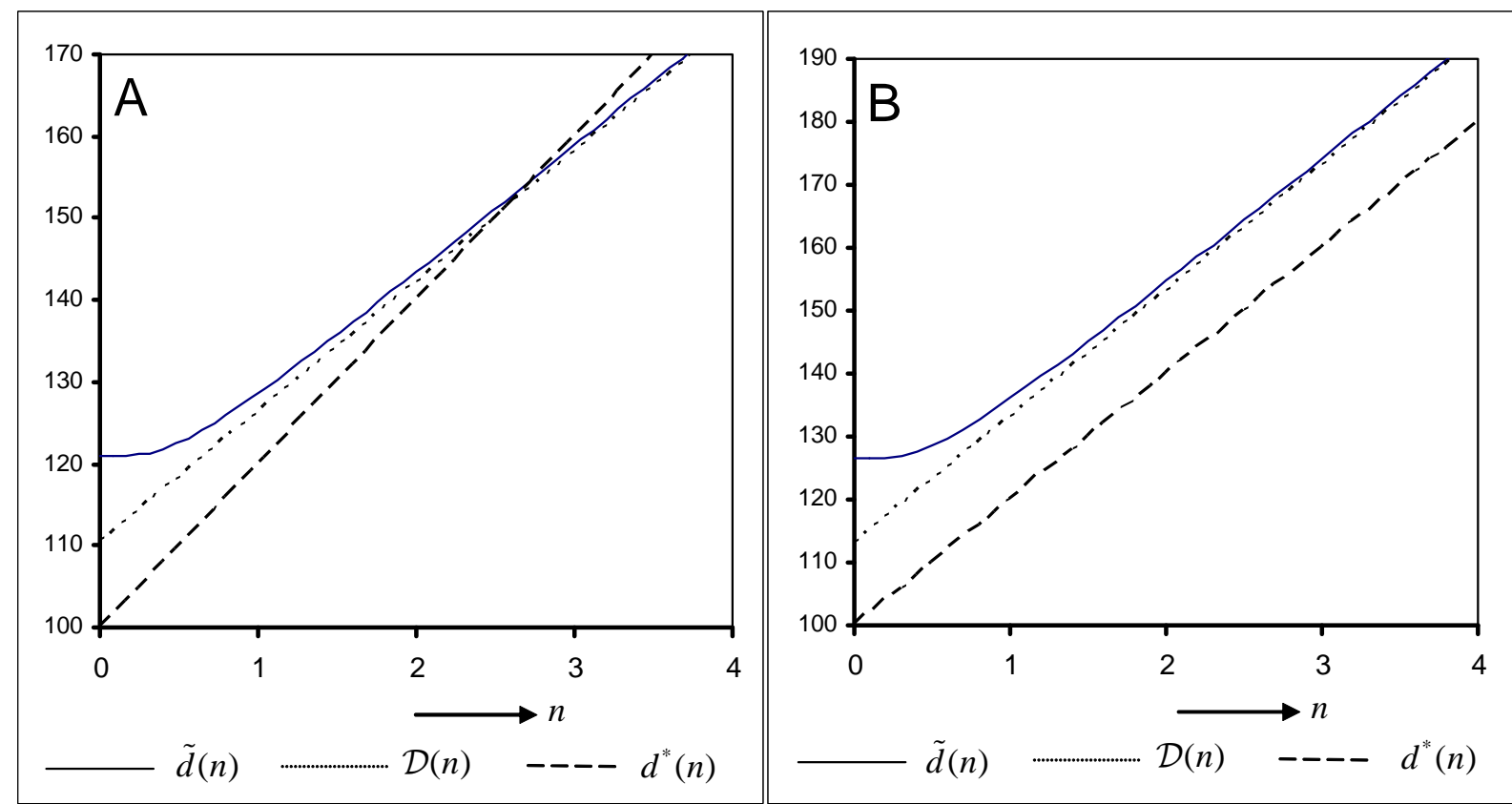

Fig. 3. Upper bound functions $d^{*}(n)(15)$ and $\widetilde{d}(n)(28)$ with its asymptote $\mathcal{D}(n)(32)$ for the data $b=80, c=100, \pi_{2}=\pi_{3}=37.5 \% ;$ A: upper quantile $d_{0.90}=121$, B: upper quantile $d_{0.90} \approx 126.425$.

After first scrutinizing the uncertainty for her market share of the new clothing line in the upcoming season (at the end of Section 2 in this paper), she decides to force ahead. Drawing from her past experience, J.D. assesses that she is $90 \%$ sure that the amount of fabric needed over the season will be above 71,000 yards, but with the same certainty level will not exceed 121,000 yards. In addition, she believes that the most likely value ranges between 80,000 and 100,000 yards. Finally, she assesses that it is 1.5 times more likely for the number of yards to fall within the estimated modal range $80,000-100,000$ than being less than 80,000 , while it is equally likely to be larger than 100,000 yards. (A somewhat optimistic assessment.). A distribution that is consistent with J.D.'s degree of belief statements above is the GTU distribution (3) in Figure 4 ( $a, b, c$ and $d$ in 1000 's). Figure 4 presents the GTU distribution which satisfies the constraints $b=80, c=100$, 
$\pi_{2} / \pi_{1}=1.5$ and $\pi_{2} / \pi_{3}=1$ and possesses lower $a_{0.10}=71$ and upper $d_{0.90}=121$ quantiles. Its mixture probabilities $\pi_{1}, \pi_{2}$ and $\pi_{3}(24)$ follow from $\pi_{2} / \pi_{1}=1.5$ and $\pi_{2} / \pi_{3}=1$. The unique tail parameters $m$ and $n$ that follow next are provided by (26) and (36). The unique lower and upper bounds $a \approx 61.085$ and $d \approx 155.135$ are given by $(27)$ and $(37)$.

J.D. evaluates from (10) and (11) a mean fabric yards needed of $94.798 \times 1000$ yards and a standard deviation equal to $18.718 \times 1000$ yards. She utilizes $(13)$ to evaluate the quantiles $x_{0.05} \approx 67.168, x_{0.50} \approx 93.333$ and $x_{0.95} \approx 128.589$ (all in 1000 yards). In other words, she believes her median fabric yards needed equals $93.333 \times 1000$ yards and its subjective $90 \%$ credibility interval equals $(67.168,128.589) \times 1000$ yards.

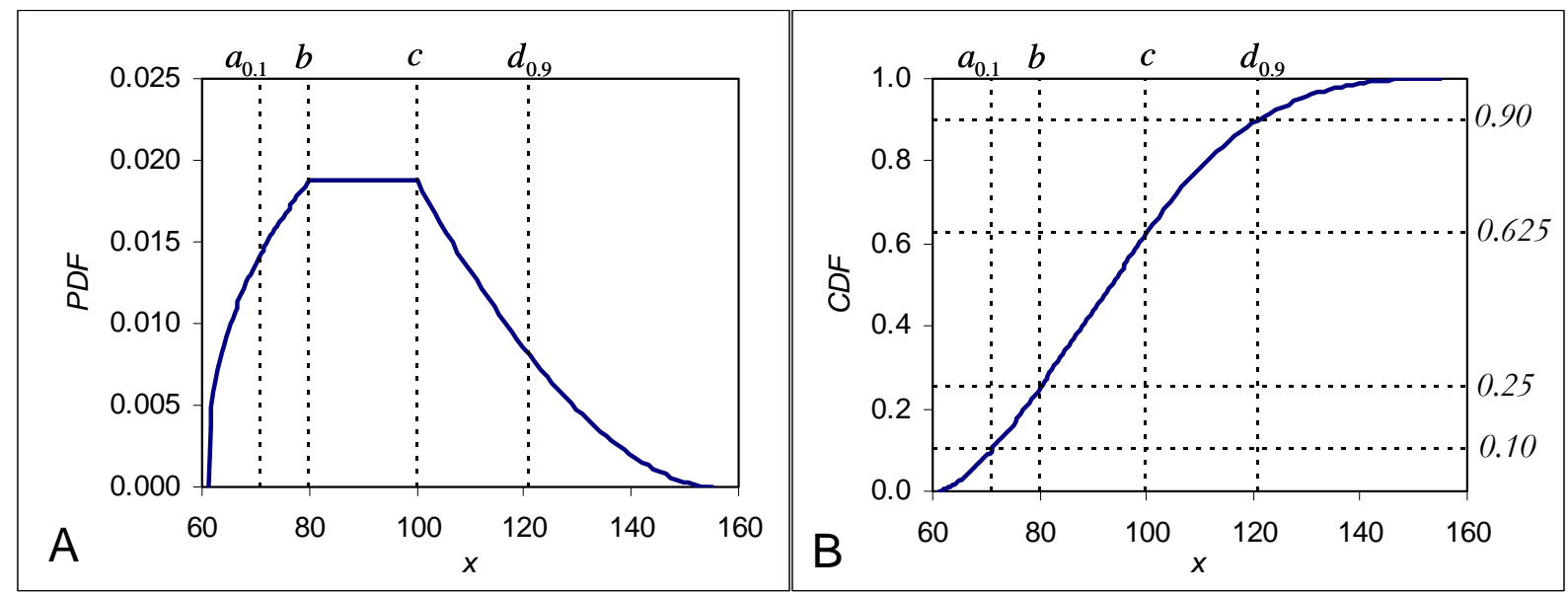

Fig. 4. GTU distribution (3) with $b=80, c=100, \pi_{2} / \pi_{1}=1.5, \pi_{2} / \pi_{3}=1$, lower quantile $a_{0.10}=71$ and upper quantile $d_{0.90}=121$. The tail parameter values $m \approx 1.419, n \approx 2.757$, and the lower $a \approx 61.085$ and upper bounds $d \approx 155.135$ were determined utilizing the equations (18) and (30).

\section{Concluding Remark}

The purpose of introducing the generalized trapezoidal-uniform distribution here is not to replace well established distributions such as the beta, triangular, asymmetric Laplace, gamma or any other appropriate distribution. It, however, does provide one additional distribution to the arsenal of available distributions to match to a substantive experts' degree of beliefs statements. 
We have described two elicitation procedures for generalized trapezoidal-uniform distributions

defined in (3) and (4). The first method assumes that the lower and upper bounds are known based on some physical boundary constraints. The second one, which is possibly more realistic albeit more involved, solves for the lower and upper bounds by eliciting a lower and upper quantiles. We believe that these elicitation procedures will facilitate an application of generalized trapezoidal-uniform distributions in problems of decision, risk and uncertainty analysis.

\section{Acknowledgement}

We are indebted to Professor Samuel Kotz for his time to provide some references, comments and suggestions in the course of the development of this paper. This paper was developed with partial support from the Decision Sciences Institute at the George Washington University.

\section{Appendix. Mathematical Properties of non-linear lower and upper bound functions involved in the elicitation procedure.}

Here we shall show that: $(i)$ the function

$$
f(n)=\alpha-\frac{x^{1 / n}}{1-x^{1 / n}}(\beta-\alpha), n \geq 0
$$

with the auxiliary parameters $x \in(0,1), \alpha$ and $\beta$ is a strictly decreasing concave (increasing convex) function in $n$ provided $\alpha<\beta(\alpha>\beta)$. Also, (ii)

$$
\lim _{n \rightarrow \infty} f(n)=\frac{\beta-\alpha}{\log (x)} n+\frac{\alpha+\beta}{2} .
$$

and finally (iii) for all $n \geq 0$ :

$$
f(n)<\frac{\beta-\alpha}{\log (x)} n+\frac{\alpha+\beta}{2} \Leftrightarrow \alpha<\beta
$$

In other words, the RHS of $(A .2)$ (which also appears in $(A .3))$ is an asymptote of the function $f(n)$ defined by $(A .1)$ and the two curves do not intersect. 
Lemma 1: The function $f(n)$ defined by $(A .1)$ is strictly decreasing and concave for $\alpha<\beta$ and is strictly increasing and concave for $\alpha>\beta$.

Proof: Assume, without loss of generality, that $\alpha<\beta$ or equivalently $\beta-\alpha>0$. (Obvious modifications can be carried out for the case $\alpha>\beta)$. Taking the first order derivative of $f(n)$ with respect to $n>0$ we have

$$
\frac{d}{d n} f(n)=(\beta-\alpha) \log (x) \frac{x^{1 / n}}{n^{2}\left(1-x^{1 / n}\right)^{2}}<0
$$

since $x \in(0,1)$. Hence, the function $f(n)$ is strictly decreasing when $\beta-\alpha>0$. Taking the second order derivative with respect to $n$ we have

$$
\frac{d^{2}}{d n^{2}} f(n)=-\frac{\log (x)}{n}\left[\frac{1}{n} \frac{1+x^{1 / n}}{1-x^{1 / n}}+\frac{2}{\log (x)}\right] \frac{d}{d n} f(n) .
$$

To prove that $f(n)$ is a concave function it is required to show that $\frac{d^{2}}{d n^{2}} f(n)<0$ for all $n>0$. Utilizing $(A .4)$ and $x \in(0,1)$ it is sufficient to prove that, for all $n>0$,

$$
\frac{1+x^{1 / n}}{1-x^{1 / n}} \times n^{-1}+\frac{2}{\log (x)}>0 \Leftrightarrow g(n)>-\frac{2}{\log (x)},
$$

where

$$
g(n)=\frac{1+x^{1 / n}}{1-x^{1 / n}} \times n^{-1}, n>0
$$

From $(A .7)$ and $x \in(0,1)$ it follows immediately that $g(n) \rightarrow \infty$ as $n \downarrow 0$ since $x^{1 / n} \downarrow 0$ as $n \downarrow 0$. Letting now $n \rightarrow \infty$, after some algebraic manipulations involving $g(n)$ and applying the L' Hopital rule we easily obtain

$$
\lim _{n \rightarrow \infty} g(n)=-\frac{2}{\log (x)}
$$

Thus to confirm the condition $(A .6)$ for all $n>0$ it is only required to prove that $g(n)$ is a strictly decreasing function.

Now denoting $m \equiv x^{1 / n} \in(0,1)$ (recall $\left.x \in(0,1)\right)$, we have for $n>0$ : 


$$
\begin{gathered}
m=\sqrt[n]{x} \Leftrightarrow n=\frac{\log (x)}{\log (m)}, \\
\frac{d m}{d n}=-\frac{\log (x)}{n^{2}} \sqrt[n]{x}=-\frac{m \log ^{2}(m)}{\log (x)} .
\end{gathered}
$$

Taking the derivative of $(A .7)$ with respect to $n$ and utilizing $(A .9)$ and $(A .10)$ we arrive at

$$
\frac{d}{d n} g(n)=\frac{d m}{d n} \frac{d}{d m} g(m)=-\frac{\log ^{2}(m)}{\{1-m\}^{2} \log ^{2}(x)} \times h(m)
$$

where

$$
h(m)=2 m \log (m)+\left(1-m^{2}\right) .
$$

Since the multiplier of $h(m)$ in the RHS of $(A .11)$ is strictly negative it follows from $(A .11)$, $m \equiv x^{1 / n}$ and $x \in(0,1)$ that

$$
\frac{d}{d n} g(n)<0 \text { for all } n>0 \Leftrightarrow h(m)>0 \text { for all } m \in(0,1) .
$$

From the definition of $h(m)$ in $(A .12), m \in(0,1)$, and noting that

$$
h(0)=1 \wedge h(1)=0
$$

and observing the form of a graph of $h(m)$ in Figure 5, we verify that indeed $h(m)>0$ for all $m \in(0,1)$ (and thus from $(A .13)$ and $(A .9)$ it follows that $g(n)$ is a strictly decreasing function). $\square$

Admittedly the plot in Figure 5 cannot serve as a formal proof. However, it is not difficult but somewhat tedious to show that the function $h(m)$ is strictly decreasing. This together with $(A .14)$ mathematically proves the RHS assertion of $(A .13)^{6}$. We invite the readers to provide an alternative (possibly simpler) proof that the function $g(n)$ defined by $(A .7)$ is strictly decreasing for $n>0$.

\footnotetext{
${ }^{6} \mathrm{~A}$ proof of $h(m)$ being strictly decreasing is available from the authors upon request.
} 


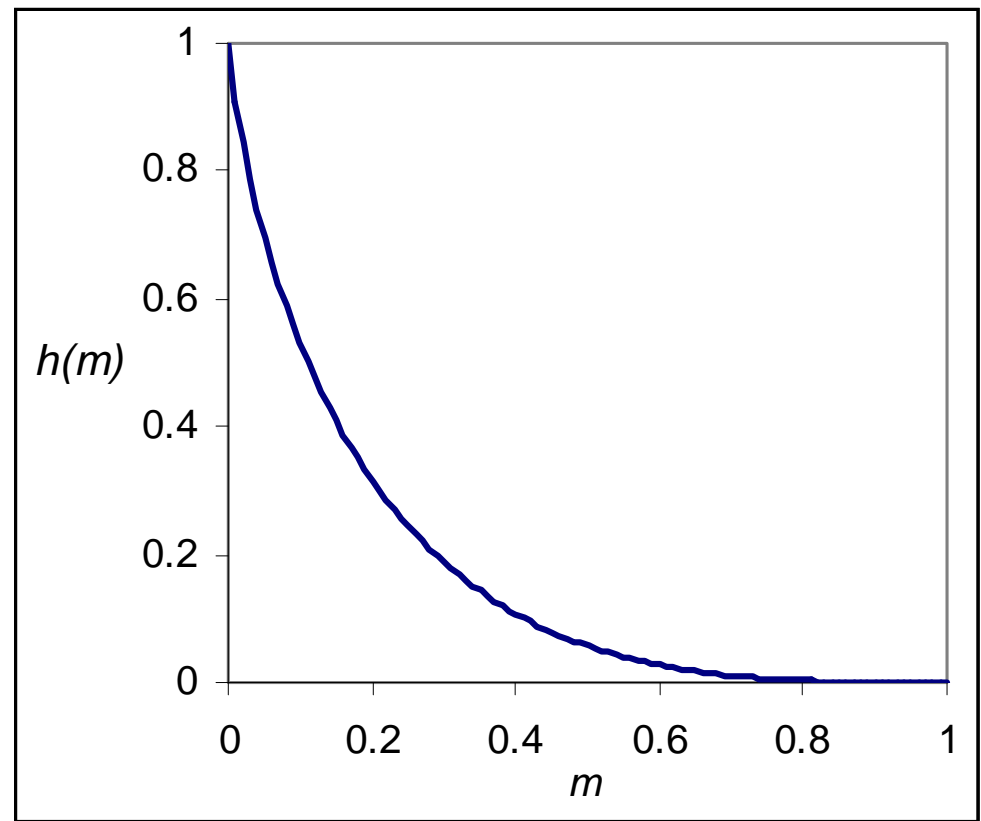

Fig. 5. A Plot of the function $h(m)$ for $m \in(0,1)$ defined by $(A .13)$.

Lemma 2: The conditions $(A .2)$ and (A.3) hold for the function $f(n)$ defined by $(A .1)$.

Proof: From the derivative $\frac{d}{d n} f(n)$ given by $(A .4)$, substituting $m=x^{1 / n}, x \in(0,1)$, and applying the L'Hopital rule twice we have

$$
\lim _{n \rightarrow \infty} \frac{d}{d n} f(n)=\frac{\beta-\alpha}{\log (x)} \lim _{m \rightarrow 1} \frac{m \log ^{2}(m)}{(1-m)^{2}}=\frac{\beta-\alpha}{\log (x)}
$$

Thus

$$
\lim _{n \rightarrow \infty} f(n)=\frac{\beta-\alpha}{\log (x)} n+\mathcal{C}
$$

where $\mathcal{C}$ is a constant. To show that the value of $\mathcal{C}=(\alpha+\beta) / 2($ see $(A .2))$ we evaluate

$\lim _{n \rightarrow \infty}\left\{f(n)-\frac{\beta-\alpha}{\log (x)} n\right\}=\lim _{n \rightarrow \infty} \frac{\left(\alpha-\beta x^{1 / n}\right) \log (x)-n(\beta-\alpha)\left(1-x^{1 / n}\right)}{\log (x)\left(1-x^{1 / n}\right)}$.

Substituting once more $m=x^{1 / n}$ we have

$$
\lim _{n \rightarrow \infty}\left\{f(n)-\frac{\beta-\alpha}{\log (x)} n\right\}=\lim _{m \rightarrow 1} \frac{(\alpha-\beta m) \log (m)-(\beta-\alpha)(1-m)}{\log (m)(1-m)} .
$$


Applying of the L'Hopital rule twice to $(A .18)$ yields

$$
\lim _{n \rightarrow \infty}\left\{f(n)-\frac{\beta-\alpha}{\log (x)} n\right\}=\frac{\alpha+\beta}{2}
$$

and the assertion $(A .2)$ is valid. Condition $(A .3)$ now follows immediately from the facts that

$$
\begin{gathered}
\lim _{n \downarrow 0} f(n)=\alpha, \\
\alpha<\frac{\alpha+\beta}{2} \Leftrightarrow \alpha<\beta
\end{gathered}
$$

and from the concavity of the function $f(n)$ iff $\alpha<\beta$ (see Lemma 1 ).

\section{References}

AbouRizk, S. M., Halpin D. W. and Wilson, J. R. (1992). Visual interactive fitting of beta distributions. Journal of Construction Engineering and Management, 117(4), 589-605.

Alpert, M. and Raiffa, H. (1982). A progress report on the training of probability assessors. In Judgment Under Uncertainty: Heuristics and Biases, D. Kahneman, Slovic, P. and Tversky, A. (Eds.), Cambridge University Press, New York, NY, pp. 294-305.

Bardosi, G. and Fodor, J. (2004). Evaluation of Uncertainty and Risks in Geology. Springer-Verlag, Berlin.

Banks, J., Carson, J.S., Nelson, B.L. and Nicol, D.M. (2005). Discrete-Event System Simulation (4th ed.), Prentice-Hall, Upper Saddle River, NJ.

Brown, S.L. (1999). An SAB Report: estimating Uncertainties in Radiogenic Cancer Risk, Washington D.C.: Science and Advisory Board, United States Environmental Protection Agency, EPA-SAB-RAC99-008.

Chen, S.J. and Hwang, C.L. (1992). Fuzzy Multiple Attribute Decision-Making: Methods and Applications, Springer-Verlag, Berlin.

Davis, C. and Sorenson K. (Editors) (1969). Handbook of Applied Hydraulics (3-rd ed.). McGraw-Hill, New York, NY.

Davidson, L.B. and Cooper D.O. (1980). Implementing effective risk analysis at Getty Oil Company. Interfaces, 10 , pp. 62-75. 
DeBrota, D. J., Roberts, S. D., Dittus, R. S. and Wilson J. R. (1989). Visual interactive fitting of bounded Johnson distributions. Simulation, 52, 199-205.

Denham, R and Mengersen, K. (2007). Geographically assisted elicitation of expert opinion for regression models. Bayesian Analysis, Vol. 2 (1), pp. 99-136.

DeWispelare, A., Herren L., Clemen, R.T. (1995). The use of probability elicitation in the high-level nuclear waste recognition program. International Journal of Forecasting, 11(1), pp. 5 - 24.

Flehinger, B.J. and Kimmel, M. (1987). The natural history of lung cancer in periodically screened population. Biometrics, 43, pp. 127-144.

Garthwaite, P.H., Kadane, J.B and O'Hagan, A. (2005). Statistical methods for eliciting probability distributions. Journal of the American Statistical Association, 100 (470), pp. 680-700.

Garvey, P.R. (2000). Probability Methods for Cost Uncertainty Analysis, a Systems Engineering Perspective. Marcel Dekker, New York, NY.

Herrerías, R. (1989). Utilización de Modelos Probabilísticos Alternativas para el Métedo PERT. Applicación al Análisis de Inversiones. Estudios de Economía Aplicada, Secretariado de Publicaciones de la Universidad de Valladolid, pp. 89-112.

Herrerías, R. and Calvete, H. (1987). Una ley de probabilidad para el estudio de los flujos de caja de una inversión. Libro Homenaje al Profesor Gonzalo Arnaiz Vellando, ICE, Madrid, pp. 279-296.

Johnson, M.A., and Taaffe, M.R. (1991). An investigation of phase-type distribution momentmatching algorithms for use in queuing models. Quening Systems, Vol. 8 (1), pp. 129-147.

Karlis, D. and Xekalaki, E. (1999). Improving the EM algorithm for mixtures. Statistics and Computing, Vol. 9 (4), pp. 303-307.

Keefer, D.L. and Bodily, S.E. (1983). Three-Point Approximations for Continuous Random Variables. Management Science, 39 (9), pp. 1086 - 1091.

Keefer, D.L. and Verdini, A.V. (1993). Better estimation of PERT activity time parameters. Management Science, 39 (9), pp. 1086 - 1091. 
Kimmel, M. and Gorlova, O.Y. (2003). Stochastic models of progression of cancer and their use in controlling cancer-related mortality. International Journal of Applied Mathematics and Computer Science, 13 (3), pp. 279-287.

Kotz, S. and Van Dorp, J.R. (2006). A novel method for fitting unimodal continuous distributions on a bounded domain. IIE Transactions, 38, pp. 421-436.

Merkhofer, M.W. (1987). Quantifying judgmental uncertainty: Methodology, experiences, and insights. IEEE Transactions of Systems, Man, and Cybernetics, SMC-17(5) 741-752.

Nakao, A. and Iwaki, M. (2000). RBS study on Na-implanted polystyrene at various doses. Applied Physics A, Materials and Science Procession, 71 (2), pp. 181-183.

O'Hagan, A. (2006). Research in Elicitation. In: Bayesian Statistics and its Applications, S. K. Upadhyay, U. Singh and D. K. Dey (eds.), pp. 375-382. Anamaya: New Delhi, 2006.

Powell, M.R. and Wilson, J.D. (1997). Risk assessment for national natural resource conservation programs, Discussion Paper 97-49. Resources for the Future, Washington, D.C.

Pouliquen, L.Y. (1970). Risk analysis in project appraisal. World Bank Staff Occasional Papers, 1. John Hopkins University Press, Baltimore, MD.

Pulkkinen, U. and Simola, K. (2000). An Expert Panel Approach to Support Risk-Informed Decision Making, Sateiluturvakeskus (Radiation and Nuclear Safety Authority of Finland STUK), Technical report STUK-YTO-TR 129, Helsinki, Finland.

Selvidge, J.E. (1980). Assessing the extremes of probability distributions by the fractile method. Decision Sciences, 11, pp. 493-502.

Sentenac, D., Shalaginov, A.N., Fera, A. and de Jue, W.H. (2000). On the instrumental resolution in X-ray reflectivity experiments. Journal of Applied Crystallography, 33, pp. 130-136.

Simpson, T. (1755). A letter to the Right Honourable George Earls of Maclesfield. President of the Royal Society, on the advantage of taking the mean of a number of observations in practical astronomy. Philosopical Transactions, 49 (1), pp. 82-93. 
Simpson, T. (1757). An attempt to show the advantage arising by taking the mean of a number of observations in practical astronomy. Miscellaneous Tracts on some curious and very interesting Subjects in Mechanics, Physical Astronomy and Speculative Mathematics, pp. 64-75.

Van Dorp, J.R. and Kotz, S. (2003). Generalized trapezoidal distributions. Metrika, 58 (1), pp. 85-97.

Wagner, M. A. F. and Wilson, J. R. (1996). Using univariate Bézier distributions to model simulation input processes. IIE Transactions, 28 (9), 699-711. 